\title{
A High-Throughput Extraction and Analysis Method for Steroidal Glycoalkaloids in Tomato
}

\author{
Michael P. Dzakovich', Jordan L. Hartman ${ }^{1}$ and Jessica L. Cooperstone ${ }^{1,2 *}$ \\ ${ }^{1}$ Department of Horticulture and Crop Science, The Ohio State University, Columbus, OH, United States, ${ }^{2}$ Department \\ of Food Science and Technology, The Ohio State University, Columbus, OH, United States
}

\section{OPEN ACCESS}

Edited by: Heiko Rischer,

VTT Technical Research Centre of Finland $L t d$., Finland

Reviewed by:

José Juan Ordaz-Ortiz, Instituto Politécnico Nacional

(CINVESTAV), Mexico Elżbieta Rytel,

Wrocław University of Environmental and Life Sciences, Poland

*Correspondence: Jessica L. Cooperstone Cooperstone.1@osu.edu

Specialty section: This article was submitted to

Plant Metabolism and Chemodiversity, a section of the journal

Frontiers in Plant Science

Received: 31 December 2019

Accepted: 14 May 2020

Published: 18 June 2020

Citation:

Dzakovich MP, Hartman JL and

Cooperstone JL (2020) A

High-Throughput Extraction and Analysis Method for Steroidal

Glycoalkaloids in Tomato.

Front. Plant Sci. 11:767.

doi: 10.3389/fpls.2020.00767
Tomato steroidal glycoalkaloids (tSGAs) are a class of cholesterol-derived metabolites uniquely produced by the tomato clade. These compounds provide protection against biotic stress due to their fungicidal and insecticidal properties. Although commonly reported as being anti-nutritional, both in vitro as well as pre-clinical animal studies have indicated that some tSGAs may have a beneficial impact on human health. However, the paucity of quantitative extraction and analysis methods presents a major obstacle for determining the biological and nutritional functions of tSGAs. To address this problem, we developed and validated the first comprehensive extraction and ultra-high-performance liquid chromatography tandem mass spectrometry (UHPLCMS/MS) quantification method for tSGAs. Our extraction method allows for up to 16 samples to be extracted simultaneously in 20 min with $93.0 \pm 6.8$ and $100.8 \pm 13.1 \%$ recovery rates for tomatidine and alpha-tomatine, respectively. Our UHPLC-MS/MS method was able to chromatographically separate analytes derived from 18 tSGA peaks representing 9 different tSGA masses, as well as two internal standards, in 13 min. Tomato steroidal glycoalkaloids that did not have available standards were annotated using high resolution mass spectrometry as well as product ion scans that provided fragmentation data. Lastly, we utilized our method to survey a variety of commonly consumed tomato-based products. Total tSGA concentrations ranged from 0.2 to $3.4 \mathrm{mg} /$ serving and represent some of the first reported tSGA concentrations in tomatobased products. Our validation studies indicate that our method is sensitive, robust, and able to be used for a variety of applications where concentrations of biologically relevant tSGAs need to be quantified.

Keywords: tomato, steroidal glycoalkaloids, Solanum lycopersicum var. cerasiforme, alpha-tomatine, tomatidine, esculeoside, lycoperoside, UHPLC-MS/MS

\section{INTRODUCTION}

Solanaceous plants produce a spectrum of cholesterol derived compounds called steroidal glycoalkaloids. While each solanaceous clade produces its own unique assortment of steroidal glycoalkaloids, these metabolites share commonality in their role as phytoanticipins and antiherbivory agents (Irving et al., 1945; Fontaine et al., 1948; Ökmen et al., 2013; Etalo et al., 2015). Tomato (Solanum lycopersicum and close relatives) is no exception, and over 100 tomato steroidal 
glycoalkaloids (tSGAs, Figure 1) have been suggested (Iijima et al., 2008, 2013). Although these compounds are typically reported as anti-nutritional (Itkin et al., 2013; Cárdenas et al., 2015, 2016; Ballester et al., 2016), other studies suggest a healthpromoting role. In fact, emerging evidence suggests that some tSGAs may play a role in positive health outcomes associated with tomato consumption (Cayen, 1971; Lee et al., 2004; Choi et al., 2012; Cooperstone et al., 2017). While these compounds continue to be evaluated both in planta and in vivo, there is a lack of quantitative and validated methods to extract and measure tSGAs from tomatoes; a critical need for additional research in this area.

Tomato steroidal glycoalkaloids are typically extracted by grinding individual samples using a mortar and pestle, or blender and then solubilizing analytes with polar solvent systems, typically methanol. This approach is time consuming because each sample is handled individually. Additionally, this technique has been used for relative profiling, and has not been evaluated for its ability to extract tSGAs quantitatively. Tomato steroidal glycoalkaloids such as alpha-tomatine, have previously been quantified using gas and liquid chromatography (Lawson et al., 1992; Rick et al., 1994; Kozukue and Friedman, 2003), as well as a number of bioassays including cellular agglutination (Schlösser and Gottlieb, 1966) and radioligand assays using radioactive cholesterol (Eltayeb and Roddick, 1984). These methods can be unreliable, suffer from poor sensitivity, have poor selectivity for different alkaloids, and are time consuming. Recent advances in analytical chemistry have enabled researchers to discover other tSGA species in tomato fruits using high resolution mass spectrometry (Iijima et al., 2008, 2013; Zhu et al., 2018), however, these methods are qualitative. A small number of quantitative methods using mass spectrometry have been developed, but only for individual or a few tSGAs (Caprioli et al., 2014; Baldina et al., 2016). Thus, there is a need to develop validated extraction and quantification methods in order to continue to study the role these compounds have in both plant and human health.

To address the lack of suitable approaches to extract and quantify tSGAs, we developed and validated a high-throughput extraction and ultra-high-performance liquid chromatography tandem mass spectrometry (UHPLC-MS/MS) method suitable for tomato and tomato-based products. Our extraction method is able to process 16 samples in parallel in $20 \mathrm{~min}$ ( $1.25 \mathrm{~min} / \mathrm{sample})$ and our UHPLC-MS/MS method can chromatographically separate, detect, and quantify 18 tSGAs (using two external and two internal standards) representing 9 different tSGA masses (Figure 2) in $13 \mathrm{~min}$ per sample. This is the first comprehensive targeted method to quantify a broad panel of tSGAs. Here, we present the experiments used to develop and validate our method as well as an application providing baseline information of tSGA concentrations in tomatoes and commonly consumed tomato products.

\section{MATERIALS AND METHODS}

\section{Chemical and Reagents}

Acetonitrile (LC-MS grade), formic acid (LC-MS grade), isopropanol (LC-MS grade), methanol (HPLC grade), and water (LC-MS grade) were purchased from Thermo Fisher Scientific (Pittsburgh, PA, United States). Alpha-tomatine ( $\geq 90 \%$ purity) and solanidine ( $\geq 99 \%$ purity) were purchased from Extrasynthese (Genay, France). Alpha-solanine ( $\geq 95 \%$ purity) and tomatidine ( $\geq 95 \%$ purity) were purchased from SigmaAldrich (St. Louis, MO, United States). Stock solutions were prepared by weighing each analyte into glass vials and dissolving into methanol prior to storage at $-80^{\circ} \mathrm{C}$. Standard curves were prepared by mixing $15 \mathrm{nmol}$ of alpha-tomatine and $1 \mathrm{nmol}$ of tomatidine in methanol. The solution was evaporated to dryness under a stream of ultra-high purity (5.0 grade) nitrogen gas. The dried residue was then resuspended in $900 \mu \mathrm{L}$ of methanol, briefly sonicated $(\sim 5 s)$, and then diluted with an additional $900 \mu \mathrm{L}$ of water. An 8-point dilution series was then prepared, and analyte concentrations ranged from $3.81 \mathrm{pmol} / \mathrm{mL}$ to $8.34 \mathrm{nmol} / \mathrm{mL}$ (11.14 femtomoles to 25 picomoles injected).

To utilize alpha-solanine and solanidine as internal standards (IS), $1.25 \mathrm{nmol}$ and $22.68 \mathrm{pmol}$ of alpha solanine and solanidine, respectively, were spiked into each vial of the alphatomatine/tomatidine external standard dilution series described above. The spike intensity of alpha-solanine and solanidine was determined by calculating the amount needed to achieve target peak areas of tSGAs typically seen in tomato samples.

\section{Sample Material}

For UHPLC-MS/MS and UHPLC-quadrupole time-of-flight mass spectrometry (UHPLC-QTOF-MS) method development experiments, 36 unique accessions of tomato including Solanum lycopersicum, Solanum lycopersicum var. cerasiforme, and Solanum pimpinellifolium were combined and pureed to create a tomato reference material expected to span the diversity of tSGAs reported in nature. For spike-in recovery experiments, red-ripe processing-type tomatoes (OH8245; courtesy of David M. Francis) were diced, mixed together by hand, and stored at $-20^{\circ} \mathrm{C}$ until analysis. Items used for the tomato product survey were purchased from supermarkets in Columbus, $\mathrm{OH}$, United States in July 2019. Three unique brands of tomato paste, tomato juice, diced tomatoes, whole peeled tomatoes, ketchup, pasta sauce, and tomato soup were analyzed for tSGAs. Additionally, four heirloom, two fresh-market, one processing, and one cherry variety of unprocessed tomatoes were also analyzed.

\section{Extraction of tSGAs}

Five grams of diced $\mathrm{OH} 8245$ tomato $( \pm 0.05 \mathrm{~g})$ were weighed in $50 \mathrm{~mL}$ falcon tubes. Five grams was selected to balance between sampling enough tissue to allow homogeneity in sampling, and to keep extraction volumes contained to a $50 \mathrm{~mL}$ tube. Two $3 / 8$ " $\times 7 / 8$ " angled ceramic cutting stones (W.W. Grainger: Lake Forest, IL, United States; Item no.: 5UJX2) were placed on top of the tomato sample and $100 \mu \mathrm{L}$ of internal standard was added, followed by $15 \mathrm{~mL}$ of methanol. Samples were then extracted for $5 \mathrm{~min}$ at $1400 \mathrm{RPM}$ using a Geno/Grinder 2010 (SPEX Sample Prep: Metuchen, NJ, United States). Sample tubes were immediately centrifuged for $5 \mathrm{~min}$ at $3000 \times g$ and $4^{\circ} \mathrm{C}$. Two $\mathrm{mL}$ aliquots of supernatant from each sample were then transferred to glass vials and diluted with $1 \mathrm{~mL}$ of water. Samples were then 


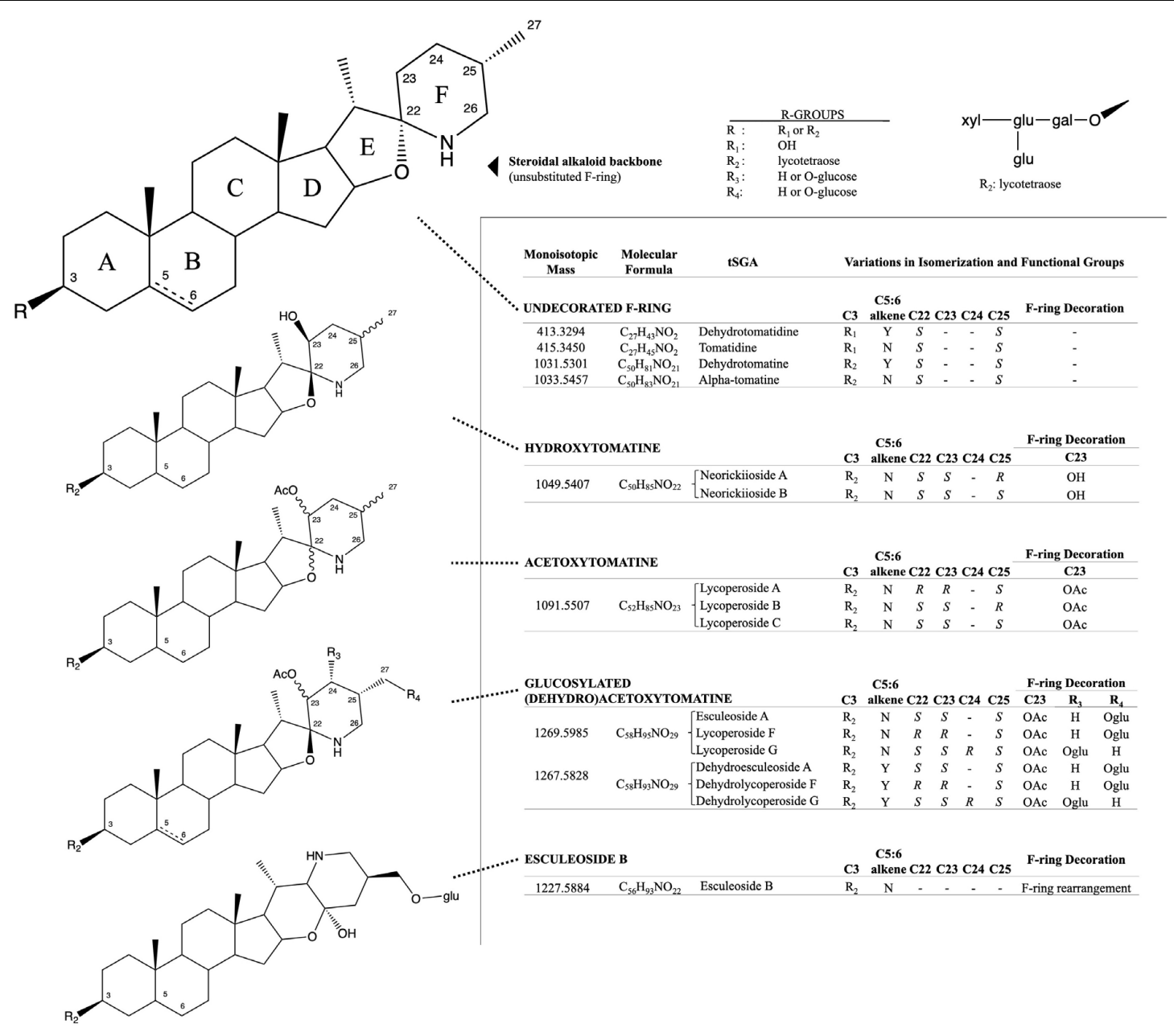

FIGURE 1 | Structural and isomeric variation in selected tomato steroidal alkaloids. Steroidal glycoalkaloids found in tomato (tSGAs) are spirosolane-type saponins with variations in a singular double-bond (C5:6), F-ring decorations (C22-C27), F-ring rearrangement (resulting in a change in stereochemistry at C22), and C3 glycosylation (typically a four-sugar tetrasaccharide, lycotetraose). The undecorated SA steroidal alkaloid backbone is shown first with relevant carbons numbered and ring names (A-F). Steroidal alkaloids were grouped based on structural similarity with bonds of varying stereochemistry denoted by wavy bonds and varying C5:6 saturation status denoted by a dashed bond. Structural variation, along with the monoisotopic mass, molecular formula, and common name are displayed alongside structures for each group. R-groups were used to denote status of $\mathrm{C} 3$ glycosylation $\left(\mathrm{R}_{1}\right.$ and $\left.\mathrm{R}_{2}\right)$ and possible positions of glucosylation on glucosylated (dehydro)acetoxytomatine $\left(R_{3}, R_{4}\right)$. All possible isomers and derivatives are not shown, just those quantitated in this method.

filtered into LC vials using a $0.22 \mu \mathrm{m}$ nylon filter (CELLTREAT Scientific Products: Pepperell, MA, United States).

Tomato products sourced from grocery stores were extracted as described above except fresh fruits of each type were blended in a coffee grinder prior to extraction. To account for differences in water content among the tomato products, $500 \mu \mathrm{L}$ aliquots from each sample were dried down under nitrogen gas, re-dissolved in $1.5 \mathrm{~mL}$ of $50 \%$ methanol, and filtered using a $0.22 \mu \mathrm{m}$ filter prior to analysis.

\section{UHPLC-MS/MS Quantification of tSGAs}

Tomato steroidal glycoalkaloids were chromatographically separated on a Waters (Milford, MA, United States) Acquity UHPLC H-Class System using a Waters C18 Acquity bridged ethylene hybrid $(\mathrm{BEH}) 2.1 \times 100 \mathrm{~mm}, 1.7 \mu \mathrm{m}$ particle size column maintained at $40^{\circ} \mathrm{C}$. The autosampler compartment was maintained at $20^{\circ} \mathrm{C}$. A gradient method with Solvent A (water $+0.1 \%(v / v)$ formic acid) and Solvent B (acetonitrile $+0.1 \%(v / v)$ formic acid) at a flow rate of $0.4 \mathrm{~mL} / \mathrm{min}$ was utilized as follows: $95 \%$ A for $0.25 \mathrm{~min}, 95 \%$ A to $80 \%$ A for $1.0 \mathrm{~min}, 80 \%$ A to $75 \%$ A for $2.5 \mathrm{~min}, 75 \%$ A held for $0.5 \mathrm{~min}$, $75 \%$ A to $68 \%$ A for $1.7 \mathrm{~min}, 38 \%$ A to $15 \%$ A for $1.7 \mathrm{~min}$, $0 \%$ A held for $3.0 \mathrm{~min}$, and back to $95 \%$ A for $2.35 \mathrm{~min}$ to reequilibrate the column. Each run lasted $13 \mathrm{~min}$ and the sample needle was washed for $10 \mathrm{~s}$ with 1:1 methanol:isopropanol before and after each injection to minimize carryover. Column eluent was directed into a Waters TQ Detector tandem mass spectrometer and source parameters and transitions can be found in Table 1. Dwell times were optimized for each analyte to allow for $12-15$ points across each peak. Quantification was carried out using 6-8 point external calibration curves, depending on the extent of linearity for a given analyte. Relative 


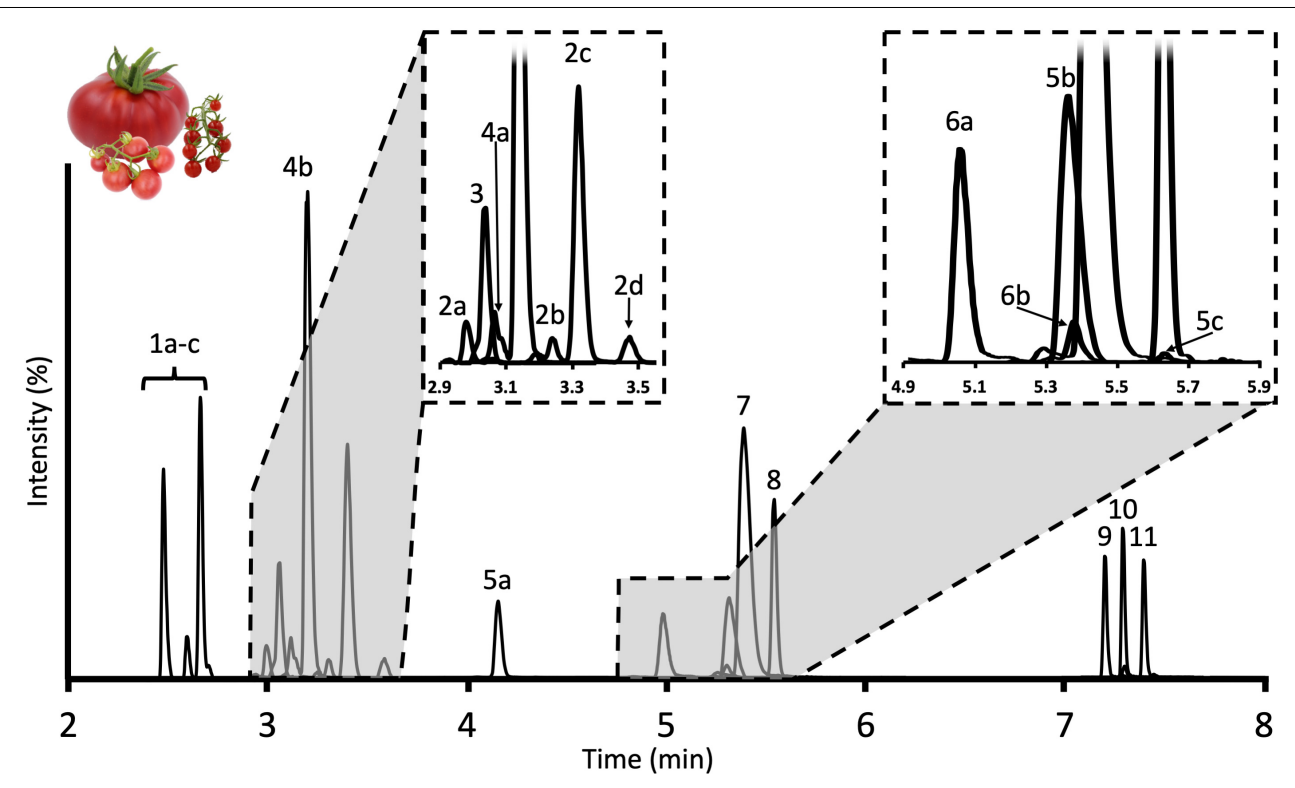

FIGURE 2 | Chromatogram of tSGAs found in red ripe tomatoes measured by our UHPLC-MS/MS method. Peaks are identified as follows: 1a-c: Esculeoside B and isomers; 2a-d: Hydroxytomatine; 3: Dehydrolycoperoside F, G, or Dehydroesculeoside A; 4a,b: Lycoperoside F, G, or Esculeoside A; 5a-c: Acetoxytomatine; 6a,b: Dehydrotomatine; 7: Alpha-tomatine; 8: Alpha-solanine; 9: Solanidine; 10: Tomatidine; 11: Dehydrotomatidine.

TABLE 1 | LC-MS/MS MRM parameters of steroidal glycoalkaloids quantified by our method.

\begin{tabular}{|c|c|c|c|c|c|}
\hline Analyte & Retention time (min) & Parent mass $[\mathrm{M}+\mathrm{H}]^{\mathrm{a}}$ & Product ions & Cone voltage (V) & $\begin{array}{c}\text { Collision energy } \\
(\mathrm{eV})\end{array}$ \\
\hline Esculeoside B & $2.55,2.67,2.74$ & 1228.6 & $254.9^{\star}, 1048.8$ & 75 & 65,40 \\
\hline Hydroxytomatine & $3.02,3.28,3.37,3.57$ & 1050.6 & $254.9,1032.7$ & 55 & 55,30 \\
\hline Dehydrolycoperoside F, G, or Dehydroesculeoside A & 3.08 & 1268.6 & $252.9^{\star}, 1208.9$ & 80 & 65,35 \\
\hline Lycoperoside F, G, or Esculeoside A & $3.11,3.26$ & 1270.6 & $1048.8,1210.9$ & 70 & 60,30 \\
\hline Acetoxytomatine $^{\mathrm{b}}(\mathrm{I})$ & 4.28 & 1092.6 & $84.7,1032.7$ & 40 & 65,35 \\
\hline Dehydrotomatine $^{\mathrm{c}}$ & $5.09,5.49$ & 1032.5 & $84.7,252.9^{\star}$ & 70 & 80,50 \\
\hline Acetoxytomatine (II) & $5.42,5.66$ & 1092.6 & $144.7,162.8$ & 40 & 50,45 \\
\hline Alpha-tomatine ${ }^{c}$ & 5.45 & 1034.6 & $84.7^{\star}, 160.8$ & 70 & 85,60 \\
\hline Alpha-solanine $e^{\mathrm{c}, \mathrm{d}}$ & 5.64 & 869.1 & $97.8^{\star}, 399.1$ & 70 & 85,65 \\
\hline Solanidine $e^{c, d}$ & 7.22 & 398.7 & $80.7,97.8^{\star}$ & 70 & 55,35 \\
\hline Tomatidine $^{\mathrm{c}}$ & 7.30 & 416.4 & $160.8,254.9$ & 50 & 30,30 \\
\hline Dehydrotomatidine $^{c}$ & 7.36 & 414.3 & $125.8,270.7$ & 40 & 30,20 \\
\hline
\end{tabular}

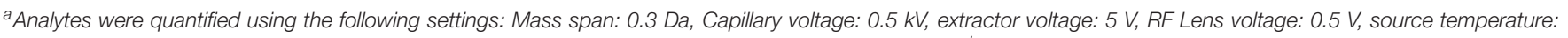

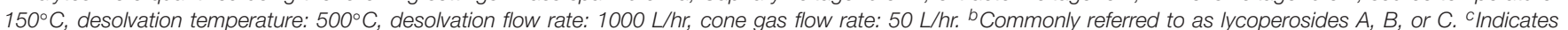
that analyte was confirmed by authentic standard. dIndicates analyte used as an internal standard. *Indicates quantifying ion; other ions used for qualifying purposes. Compounds with no indicated quantifying ion were quantified using the sum of both MRM transitions.

quantification was used for tSGAs (quantified using alphatomatine) and their aglycones (quantified using tomatidine) that did not have commercially available standards. Additionally, signals were normalized to alpha-solanine and solanidine for glycosylated and aglycone analytes, respectively, to correct for instrument variability.

\section{UHPLC-QTOF/MS Confirmation of tSGA Identities}

We verified the identities of our tSGA analytes using an Agilent 1290 Infinity II UHPLC coupled with an Agilent
6545 QTOF-MS. Identical column and chromatographic separation conditions were used as described above for our MS/MS method. The QTOF-MS used an electrospray ionization source operated in positive mode and data were collected from 50 to $1700 \mathrm{~m} / z$ for both full-scan and MS/MS experiments. Gas temperature was set to $350^{\circ} \mathrm{C}$, drying gas flow was $10 \mathrm{~L} / \mathrm{min}$, nebulizer gas flow was $10 \mathrm{~L} / \mathrm{min}$, nebulizer was $35 \mathrm{psig}$, and sheath gas flow and temperature was $11 \mathrm{~L} / \mathrm{min}$ and $375^{\circ} \mathrm{C}$, respectively. For MS/MS experiments on the QTOF-MS, identical parameters were used except for the selection of tSGA masses of interest and a 2-min retention time window around each analyte 
to maximize duty cycle of the instrument. Collision energy for all tSGAs was set to $70 \mathrm{eV}$ and all aglycones were fragmented with $45 \mathrm{eV}$.

\section{Limit of Detection and Limit of Quantification}

Limit of detection and LOQ were calculated using six replicates of the lowest concentration standard curve calibrant sample (3.81 and 0.254 femtomoles on column for alpha-tomatine and tomatidine, respectively), and determining their signal to noise ratios. Moles on column at $3 / 1$ and $10 / 1$ signal to noise were then determined for alpha-tomatine and tomatidine to calculate LOD and LOQ.

\section{Spike Recovery Experiments}

Ten, $5 \mathrm{~g}( \pm 0.01 \mathrm{~g})$ replicates of diced OH8245 processing tomatoes were weighed into $50 \mathrm{~mL}$ falcon tubes. Five samples were extracted as outlined previously with the addition of a $100 \mu \mathrm{L}$ methanolic solution containing $1.67 \mathrm{nmol}$ of alpha-tomatine, $1.25 \mathrm{nmol}$ of alpha solanine, $12.4 \mathrm{pmol}$ of tomatidine, and $22.68 \mathrm{pmol}$ of solanidine (spiked tomato) while another five samples were extracted without IS solution (non-spiked tomato; $100 \mu \mathrm{L}$ of methanol used in its place). The IS was allowed to integrate into the sample matrix for $30 \mathrm{~min}$. Another set of five samples were prepared by substituting tomato with $5 \mathrm{~mL}$ of water and extracted with the addition of $100 \mu \mathrm{L}$ of the methanolic IS solution mentioned previously (spiked mock sample). Percent recovery was estimated using the following equation:

$$
\text { Recovery }(\%)=\frac{\text { Spiked Tomato }}{\text { Non }- \text { spiked Tomato }+ \text { Spiked Mock Sample }}
$$

\section{Intra/Interday Variability Experiments}

Eight OH8245 tomato fruits were blended together and $5 \mathrm{~g}$ aliquots $( \pm 0.05 \mathrm{~g})$ were distributed among $18,50 \mathrm{~mL}$ tubes, and frozen at $-20^{\circ} \mathrm{C}$. Over 3 days, six tubes were randomly selected from the freezer each day and tSGAs were extracted and quantified as outlined above by a single individual. Intraday variability was determined by computing the coefficient of variation for an analyte within a day. Interday variability was calculated by taking the coefficient of variation of all samples run over the 3-day period.

\section{Autosampler Stability Experiments}

A quality control sample containing multiple tomato species, as described above was extracted with the addition of $100 \mu \mathrm{L}$ of IS solution as outlined previously. Over a period of $12 \mathrm{~h}$, the quality control sample was injected and analyzed by UHPLC-MS/MS at hourly intervals. The vial cap was replaced after each injection to prevent sample evaporation between injections and the autosampler compartment was maintained at $20^{\circ} \mathrm{C}$.

\section{RESULTS AND DISCUSSION}

\section{Development of High-Throughput Extraction and UHPLC-MS/MS Quantification Methods Development of High-Throughput Extraction Method} Generally, tomato samples are pulverized in a mortar in the presence of liquid nitrogen or homogenized using a blender prior to extracting tSGAs. Tomato steroidal glycoalkaloids are considered semi-polar metabolites and are typically extracted via physical disruption in a methanolic solvent system (Moco et al., 2006; Iijima et al., 2008, 2013; Mintz-Oron et al., 2008; Ballester et al., 2016). Current methods are time consuming since each sample needs to be processed individually. Our protocol features a combined homogenization/extraction step using a Geno/Grinder system that can process up to 16 samples at once. Given a 5-min homogenization/extraction, 5-min centrifugation, and an approximately 10-min dilution/filtration step, our extraction method can process 16 samples every $20 \mathrm{~min}$ (1.25 $\mathrm{min} / \mathrm{sample})$ making it ideal for screening large tomato populations or large sample sets of tomato products. Moreover, the tomato sample is able to stay frozen until the extraction begins which prevents potential enzymatic modification and degradation of analytes.

\section{Selection of Precursor lons}

Over 100 tSGAs have been tentatively identified in tomato using high-resolution mass spectrometry and some with MS/MS fragmentation (Iijima et al., 2008, 2013; Zhu et al., 2018). However, we do not know the specific concentrations of tSGA accumulating in fruits. To study tSGAs further, quantitative analysis methods are necessary. In order to maximize the amount of tSGAs detected and separated in our method, we first compiled a target list of biologically relevant tSGAs by surveying the literature (Fujiwara et al., 2004; Iijima et al., 2009; Alseekh et al., 2015; Cichon et al., 2017; Cooperstone et al., 2017; Zhu et al., 2018; Hövelmann et al., 2019). Tomato steroidal glycoalkaloid species were prioritized based on their perceived abundance in the tomato clade, previous structural characterization, and having an established record of being impacted by or a part of biological processes such as ripening or plant defense. Using this process, 18 masses covering at least 25 different tSGA species were selected for chromatographic separation and quantification.

A $50 \%$ aqueous methanolic extract from a reference material comprised of red-ripe Solanum lycopersicum, Solanum lycopersicum var. cerasiforme, and Solanum pimpinellifolium fruits was used for method development on a Waters Acquity UHPLC H-Class System connected to a TQ Detector triple quadrupole mass spectrometer with electrospray ionization operated in positive ion mode. A gradient progressing from 5 to $100 \%$ acetonitrile over $15 \mathrm{~min}$ run on a Waters $2.1 \times 100 \mathrm{~mm}$ ( $1.7 \mu \mathrm{m}$ particle size) column at $0.4 \mathrm{~mL} / \mathrm{min}$ was used to separate as many potential analytes as possible. Selected Ion Recordings (SIRs) of masses of interest were utilized to identify potential tSGA species. Since only two tomato alkaloids of interest are available commercially (alpha-tomatine and tomatidine), elution 
order, accurate mass, and fragmentation patterns were used to assign identity all other tSGAs. Source parameters of the MS were then adjusted to the maximize signal of both identified and tentatively identified tSGAs Those tSGAs which were readily detectable in our pooled tomato quality control samples were used in our final method. While studied more extensively than many other tSGAs, we were not able to detect and quantify beta-, gamma-, and delta-tomatine in our reference material, and thus they are not included in our panel.

\section{Use of Internal Standards}

We tested three, commercially available potato-derived alkaloids for their suitability as internal standards to correct for inter and intraday variability created in the MS. Alpha-solanine, alpha-chaconine, and solanidine (aglycone of alpha-solanine) were selected based on their similarity in structure, ionization efficiencies and retention times to tomato-derived alkaloids. However, alpha-chaconine was excluded due to co-elution with alpha-tomatine. We determined $1.25 \mathrm{nmol}$ and $22.68 \mathrm{pmol}$ of alpha solanine and solanidine, respectively, should be added to each sample (41.7 femtomoles of alpha solanine and 0.756 femtomoles of solanidine on column) to achieved comparable peak areas to those observed for tSGAs and their aglycones such as tomatidine and dehydrotomatidine (Figure 2). Alphasolanine and solanidine multiple reaction monitoring (MRMs) experiments were then optimized in tandem with tSGAs of interest as follows.

\section{Optimization of MS Parameters}

Desolvation temperature, desolvation gas flow rate, and cone voltage were experimentally optimized. All other source parameters remained at their recommended default settings and are reported in the footer of Table 1. For all experiments, vial caps were replaced after each injection to prevent any possible effects from evaporation through the pierced septa. To optimize the desolvation temperature, a 50\% aqueous methanolic solution of alpha-tomatine and tomatidine was injected and desolvation temperatures ranging from 350 to $500^{\circ} \mathrm{C}$ at $25^{\circ} \mathrm{C}$ increments were tested. A $500^{\circ} \mathrm{C}$ desolvation temperature resulted in the highest signal. Desolvation gas flow was tested in a similar manner starting from 600 to $1000 \mathrm{~L} / \mathrm{hr}$ in $100 \mathrm{~L} / \mathrm{hr}$ increments. Likewise, the $1000 \mathrm{~L} / \mathrm{h}$ flow rate resulted in the most signal for both analytes. Alpha-tomatine and tomatidine were used in these experiments because of their commercial availability, their structural similarity to other tSGAs of interest, and their intended use for relative quantification of all other tSGAs and their aglycones. Finally, cone voltage was optimized by injecting a $50 \%$ aqueous methanolic extract of our tomato reference material and measuring the signal of each SIR. Cone voltages ranged from 20 to $90 \mathrm{~V}$ and successive injections were made in $5 \mathrm{~V}$ increments. Optimal cone voltages were specific to each mass and are notated in Table 1. With source parameters set to optimize the signal of all precursor ions of interest, product ion scans were then conducted to tentatively identify tSGAs and aid in the development of MRM experiments, which were ultimately used for quantification.
Since each SIR yielded multiple peaks, information from product ion scans was leveraged to determine if each peak was actually a tSGA. Product ion scan experiments were created for each mass of interest and multiple collision energies $(20,45$, and $65 \mathrm{eV}$ ) were tested. The resulting spectra generated for each peak allowed us to eliminate peaks that were isobaric with tSGAs of interest, but had product ions inconsistent with proposed structures. Masses such as 255 and $273 \mathrm{~m} / z$ were particularly useful in identifying alkaloids as they are likely derived from the fragmentation of the steroidal backbone characteristic of all tSGAs (Supplementary Material) and have been previously reported in the literature (Iijima et al., 2013; Caprioli et al., 2014; Cichon et al., 2017; Sonawane et al., 2018; Zhu et al., 2018). Additionally, tSGAs with the prefix "dehydro" exhibit a desaturation on the $\mathrm{B}$ ring of the steroidal backbone between carbons 5 and 6 (Ono et al., 1997; Itkin et al., 2011; Iijima et al., 2013; Sonawane et al., 2018). We observed that common fragments derived from the steroidal backbone of these alkaloids, such as 253 and 271, were accordingly $2 \mathrm{~m} / z$ less than their saturated counterparts. The 273 and 255 fragments correspond to the A-D rings of the steroidal backbone and its corresponding water loss product (Sonawane et al., 2018). Elution order of analytes was used to help tentatively identify tSGAs detected in our reference sample based on previous reports (Alseekh et al., 2015; Zhu et al., 2018). Multiple collision energies allowed us to select product ions that were abundant and consistently produced under different conditions. These product ions then became candidate ions for MRM development.

MRM experiments allowed us to confidently detect and quantify tSGAs of interest and increase sensitivity by minimizing interference of co-eluting compounds. We created MRM experiments for each mass using optimized source conditions and four product ions with the highest signal/noise ratio. Initially, our $50 \%$ aqueous methanolic reference sample extract was injected and each transition was tested at $5 \mathrm{eV}$. The experiments were rerun at increasing collision energies at $15 \mathrm{eV}$ increments up to $95 \mathrm{eV}$. Afterward, a $20 \mathrm{eV}$ window broken into $5 \mathrm{eV}$ increments was determined for each transition and the experiments were rerun. Optimized MRMs are displayed in Table 1. To maximize duty cycle, two transitions with the best signal to noise ratio were retained. The gradient was then optimized to chromatograph each analyte. All tSGAs were quantified using a standard curve generated with alpha-tomatine while aglycone species used tomatidine. Due to the structural similarity among tSGA species quantified in our method, we hypothesize that ionization efficiencies will be similar amongst our analytes. Lastly, MRMs were developed for the potato derived alkaloids alpha-solanine and solanidine used as IS. These IS allowed us to correct for instrument derived variability that normally occurs with mass spectrometers.

\section{Development of Chromatographic Gradient}

Method development related to the MS was initially carried out using a simple 13-min gradient outlined above. While this run time is shorter than many of the previously published studies characterizing tSGAs using high-resolution MS (Iijima et al., 2008, 2013; Zhu et al., 2018) we aimed to create a 
more efficient method that would be able to accommodate large sample sets. Of the two columns tested (Waters C18 Acquity bridged ethylene hybrid (BEH) $2.1 \times 100 \mathrm{~mm}$, $1.7 \mu \mathrm{m}$ and Waters C18 Acquity high strength silica (HSS) $2.1 \times 100 \mathrm{~mm}, 1.8 \mu \mathrm{m})$, the BEH column was able to better resolve analytes of interest with a particular benefit observed in the nonpolar aglycone steroidal alkaloids. We adjusted our gradient conditions in such a way that all separation of analytes occurred within a 6-min window with an additional $5 \mathrm{~min}$ devoted to cleaning and requilibrating the column to reduce carryover (Figure 2). Additionally, the needle wash was set to rinse the needle and injection port for $10 \mathrm{~s}$ before and after an injection with 1:1 methanol:isopropanol to further reduce carryover. We observed multiple peaks for many of our masses indicating the presence of multiple isobaric tSGAs (likely including structural isomers) (Figure 2). In the case of esculeoside $\mathrm{B}$, multiple diastereomers have been previously reported in tomato products which explains our observation of multiple peaks for this analyte (Manabe et al., 2013; Nohara et al., 2015; Hövelmann et al., 2019). Validation experiments, including confirmation of peak identities using high-resolution mass spectrometry, were next carried out using the finalized chromatographic gradient.

\section{Validation of Extraction and UHPLC-MS/MS Methods Confirmation of Analytes Using High-Resolution}

High resolution mass spectrometry was used to confirm the identities of analytes quantified by our UHPLC-MS/MS method. We transferred our method to an Agilent 1290 Infinity II connected to an Agilent 6545 QTOF and profiled tSGAs both in high resolution full scan mode $(50-1700 \mathrm{~m} / z)$ and through targeted fragmentation experiments. Both types of experiments were consistent with our identities of all tSGAs and aglycones in our UHPLC-MS/MS method (Table 2). Retention times differed slightly between the UHPLC-MS/MS method and the UHPLCQTOF-MS experiments due to differences in dead volume between the two instruments. However, relative elution order remained the same.

Targeted MS/MS experiments using UHPLC-QTOF-MS allowed us to determine common spectral characteristics for each tSGA (Figure $\mathbf{1}$ and Supplementary Material). Using commercially available alpha-tomatine and tomatidine and exploiting the presence of dehydrotomatine and dehydrotomatidine (tomatidenol) as impurities within these standards, we were able to collect MS/MS fragmentation data on these four analytes. We found that all tSGAs and aglycones fragmented in predictable ways that allow for identification. Common masses produced by each tSGA in our method can be found in Table 2 . These data allow us to tentatively identify all analytes in our UHPLC-MS/MS with a high degree of confidence.

\section{LOD and LOQ}

Previous chromatography-based methods to quantify both potato and tSGAs relied on photodiode array detectors set to 208 nm (Kozukue and Friedman, 2003; Kozukue et al., 2004;
Tajner-Czopek et al., 2014; Del Giudice et al., 2015). Given that the molar extinction coefficient for alpha-tomatine is only $5000 \mathrm{M}^{-1} \mathrm{c}^{-1}$, (Keukens et al., 1994), photodiode array detectors are often not sensitive enough for detecting low quantities of these compounds, nor distinguishing between different alkaloids. Moreover, photodiode array detectors are often set to $208 \mathrm{~nm}$ to quantify tSGAs which is a non-specific wavelength where many compounds (including mobile phases) can absorb light (Friedman and Levin, 1992, 1998; Keukens et al., 1994). Mass spectrometers offer substantial gains in sensitivity through the use of MRM experiments and the ability to differentiate numerous analytes in a single run. Our UHPLC-MS/MS method for quantifying tSGAs was able to detect and quantify alphatomatine and tomatidine in the low femtomole-on-column range (Table 3). Given our extraction method, tSGAs could be present in picomolar concentrations in tomato and still be quantified. Previously reported limits of quantification for alphatomatine range from $0.005 \mathrm{mg} / \mathrm{kg}$ (estimated to be $0.5 \mu \mathrm{g}$ in a standard 100 g tomato) (Caprioli et al., 2014). Given our reported LOQ of 1.10 femtomoles injected, we estimate that 681 picograms of alpha-tomatine can be quantified in tomatoes making our method almost three orders of magnitude more sensitive. This sensitivity could also be useful for situations when fruit quantity is lacking.

\section{Spike Recovery}

Spike addition experiments were conducted to assess the performance of our high-throughput extraction method. Both tomato and potato derived external alkaloid standards were used to determine if our chosen internal standards would behave similarly to analytes native to tomato. Tomato alkaloids alpha-tomatine $(100.8 \% \pm 13.1)$ and tomatidine $(93 \% \pm 6.8)$ as well as the potato-derived internal standards alpha solanine $(94.3 \% \pm 3.4)$ and solanidine $(99.7 \% \pm 7.1)$ were efficiently extracted using our method (Table 3). These data indicate that our method is able to effectively extract aglycone and glycosylated steroidal alkaloid species from tomato and our internal standards extract similarly to native analytes.

\section{Intra/Interday Variability}

Experiments to determine intra/interday variability were conducted to determine analytical variability in our extraction and analysis methods. A single operator extracted six tomato samples and analyzed them by UHPLC-MS/MS. This experiment was repeated twice more by the same operator. Our data indicate that our methods are reliable with most analytes having coefficient of variations for both intra and interday variability below 5\% (Table 4). As expected, interday variability was higher than intraday variability for all analytes reflecting day-to-day variability in the MS.

\section{2-h Stability Experiment}

Tomato phytochemicals typically analyzed, such as carotenoids, are subject to oxidation and need to be run in small batches to minimize experimental error due to degradation (Kopec et al., 2012). However, relatively little is known about the 
TABLE 2 | UHPLC-QTOF-MS confirmation of tSGA identities.

\begin{tabular}{|c|c|c|c|c|c|c|}
\hline $\begin{array}{l}\text { Tentative } \\
\text { identification }\end{array}$ & Molecular formula & Retention time (min) & Monoisotopic mass & Observed mass $[\mathrm{M}+\mathrm{H}]$ & Mass error ( $\Delta \mathrm{ppm})$ & Common MS/MS fragments ${ }^{a}$ \\
\hline \multirow[t]{3}{*}{ Esculeoside B } & $\mathrm{C}_{56} \mathrm{H}_{93} \mathrm{NO}_{28}$ & 2.24 & 1227.5884 & 1228.5989 & 2.20 & $\begin{array}{l}\text { 1048.5380, 273.2120, 255.2016, 163.0509, } \\
\text { 145.0404, 85.0205 }\end{array}$ \\
\hline & & 2.34 & & 1228.5967 & 0.41 & \\
\hline & & 2.45 & & 1228.5966 & 0.33 & \\
\hline \multirow[t]{4}{*}{ Hydroxytomatine } & $\mathrm{C}_{50} \mathrm{H}_{83} \mathrm{NO}_{22}$ & 2.84 & 1049.5407 & 1050.5500 & 1.43 & $\begin{array}{l}\text { 1032.5385, 273.2213, 255.2203, 161.1318, } \\
\text { 145.0489, 85.0279 }\end{array}$ \\
\hline & & 3.22 & & 1050.5513 & 2.67 & \\
\hline & & 3.29 & & 1050.5506 & 2.00 & \\
\hline & & 3.50 & & 1050.5501 & 1.52 & \\
\hline $\begin{array}{l}\text { Dehydrolycoperoside F, } \\
\text { G, or } \\
\text { Dehydroesculeoside A }\end{array}$ & $\mathrm{C}_{58} \mathrm{H}_{93} \mathrm{NO}_{29}$ & 2.41 & 1267.5828 & 1268.5930 & 1.89 & $\begin{array}{l}\text { 1208.5714, 1046.5175, 271.2054, 253.1951, } \\
163.0600,85.0284\end{array}$ \\
\hline \multirow[t]{2}{*}{$\begin{array}{l}\text { Lycoperoside F, G, or } \\
\text { Esculeoside A }\end{array}$} & $\mathrm{C}_{58} \mathrm{H}_{95} \mathrm{NO}_{29}$ & 2.44 & 1269.5985 & 1270.6076 & 1.02 & $\begin{array}{l}1210.5900,1048.5324,273.2213,255.2108, \\
163.0600,85.0285\end{array}$ \\
\hline & & 3.06 & & 1270.6095 & 2.52 & \\
\hline Acetoxytomatine (I) & $\mathrm{C}_{52} \mathrm{H}_{85} \mathrm{NO}_{23}$ & 4.22 & 1091.5507 & 1092.5614 & 2.65 & $\begin{array}{l}\text { 1032.5386, 273.2216, 255.2112, 161.1326, } \\
145.0497,85.0287\end{array}$ \\
\hline \multirow[t]{2}{*}{ Dehydrotomatine $^{b}$} & $\mathrm{C}_{50} \mathrm{H}_{81} \mathrm{NO}_{21}$ & 4.99 & 1031.5301 & 1032.5388 & 0.87 & $\begin{array}{l}\text { 1014.5274, } 271.2054,253.1951,145.0495 \\
85.0284,57.0337\end{array}$ \\
\hline & & 5.20 & & 1032.5373 & 0.58 & \\
\hline \multirow[t]{2}{*}{ Acetoxytomatine (II) } & $\mathrm{C}_{52} \mathrm{H}_{85} \mathrm{NO}_{23}$ & 5.32 & 1091.5507 & 1092.5619 & 3.11 & $\begin{array}{l}\text { 1032.5404, 273.2216, 255.2114, 161.1328, } \\
\text { 145.0499, 85.0288 }\end{array}$ \\
\hline & & 5.39 & & 1092.5608 & 2.11 & \\
\hline Alpha-tomatine ${ }^{b}$ & $\mathrm{C}_{50} \mathrm{H}_{83} \mathrm{NO}_{21}$ & 5.35 & 1033.5457 & 1034.5557 & 2.13 & $\begin{array}{l}\text { 1016.5449, 416.3523, 273.2217, } 255.2112 \\
\text { 145.0498, } 85.0287\end{array}$ \\
\hline Tomatidine $^{b}$ & $\mathrm{C}_{27} \mathrm{H}_{45} \mathrm{NO}_{2}$ & 6.95 & 415.3450 & 416.3531 & 0.72 & $\begin{array}{l}\text { 398.3414, 273.2208, 255.2101, 161.1318, } \\
126.1271,81.0693\end{array}$ \\
\hline Dehydrotomatidine ${ }^{b}$ & $\mathrm{C}_{27} \mathrm{H}_{43} \mathrm{NO}_{2}$ & 6.98 & 413.3294 & 414.3371 & 0.24 & $\begin{array}{l}\text { 396.3260, 271.2053, 253.1949, 161.1322, } \\
126.1275,81.0695\end{array}$ \\
\hline
\end{tabular}

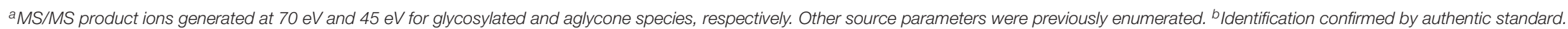


TABLE 3 | Extraction efficiency of commercially available tSGAs and potato-derived internal standards.

\begin{tabular}{|c|c|c|c|c|}
\hline Analyte & Sample size & $\begin{array}{c}\text { Extraction } \\
\text { efficiency }(\%)\end{array}$ & $\begin{array}{c}\text { LOQ } \\
\text { (femtomoles } \\
\text { injected) }\end{array}$ & $\begin{array}{c}\text { LOD } \\
\text { (femtomoles } \\
\text { injected) }\end{array}$ \\
\hline Alpha-tomatine & $n=6$ & $100.8 \pm 13.1$ & 1.0988 & 0.3296 \\
\hline Alpha-solanine ${ }^{a}$ & $n=6$ & $94.3 \pm 3.4$ & $N / A^{b}$ & $\mathrm{~N} / \mathrm{A}$ \\
\hline Tomatidine & $n=6$ & $93.0 \pm 6.8$ & 0.3354 & 0.1006 \\
\hline Solanidine ${ }^{a}$ & $n=6$ & $99.7 \pm 7.1$ & $\mathrm{~N} / \mathrm{A}$ & $\mathrm{N} / \mathrm{A}$ \\
\hline
\end{tabular}

a Analyte used as an internal standard with no calibration curve. ${ }^{b}$ Not applicable due to its use as an internal standard.

TABLE 4 | Intraday and interday coefficient of variation values for analytes quantified by our UHPLC-MS/MS method.

\begin{tabular}{lcc}
\hline Analyte & $\begin{array}{c}\text { Intraday coefficient } \\
\text { of variation (\%) }\end{array}$ & $\begin{array}{c}\text { Interday coefficient } \\
\text { of variation }^{\mathbf{( \%}} \mathbf{~}^{\mathbf{b}}\end{array}$ \\
\hline Esculeoside B & 4.46 & 6.84 \\
Hydroxytomatine & 4.00 & 5.60 \\
Dehydrolycoperoside & 8.42 & 8.03 \\
F, G, or & & \\
Dehydroesculeoside & & 4.21 \\
A & 3.35 & 3.89 \\
Lycoperoside F, G, & 3.56 & 7.11 \\
or Esculeoside A & 4.25 & 7.70 \\
Acetoxytomatine (I) & 7.57 & 6.42 \\
Dehydrotomatine & 3.92 & 13.73 \\
Acetoxytomatine (II) & 11.78 & 13.61 \\
Alpha-tomatine & 11.69 & \\
Tomatidine & & \\
Dehydrotomatidine & &
\end{tabular}

a Average coefficient of variation within a day of six samples extracted and run by a single operator. The experiment was repeated over 3 days. ${ }^{b}$ Average coefficient of variation over a 3-day period of 18 samples extracted and run by a single operator.

stability of tSGAs compared to the above phytochemical classes. We hypothesized that due to the known heat stability of chemically analogous potato steroidal glycoalkaloids, extracted tSGAs would be stable over time. A 12-h stability study demonstrated that both alpha-tomatine and tomatidine did not degrade over time in an autosampler maintained at 20 ${ }^{\circ} \mathrm{C}$. This stability enables large batching of analysis of tSGAs in $\sim 50$ samples at a time, and 100 tomato extracts per day. Analysis of large numbers of samples is critical for plant breeders and large-scale diversity analyses. While there is currently no published literature investigating the stability of tSGAs, some data exists in chemically analogous potato glycoalkaloids. Often, potato glycoalkaloids are extracted at $100^{\circ} \mathrm{C}$ temperatures to disrupt cell walls and otherwise weaken the sample matrix (Rodriguez-Saona et al., 1999) and processing studies have shown that these compounds are stable up to $180^{\circ} \mathrm{C}$ (Chungcharoen, 1988). Therefore, tSGAs may also have similar heat tolerance attributes and we speculate that these analytes may remain unchanged in autosamplers well beyond the 12-h time period we tested.

\section{Application of Extraction and UHPLC-MS/MS Method Grocery Store Survey}

To test our extraction and quantification method, we surveyed several commonly consumed tomato-based products available at grocery stores. The purpose was twofold: to test applicability of or method, and to report comprehensive and quantitative values of tSGAs in commonly consumed tomato products. These products included an assortment of fresh tomatoes, ketchup, pasta sauce, pizza sauce, tomato soup, tomato paste, tomato juice, and whole peeled tomatoes (Table 5). Values are reported per serving to normalize between tomato products subjected to varying degrees of concentration. While there are some reports of tSGA concentrations in fresh tomatoes using modern methods (Baldina et al., 2016), concentrations in tomato-based products are not well reported in the literature. Though alpha-tomatine degrades during ripening (Kozukue and Friedman, 2003), other tSGAs, including lycoperosides F, G and esculeoside A, and esculeoside B increase during this period, keeping total concentrations of tSGA roughly constant (Yamanaka et al., 2009). We found that tSGAs varied depending on type of product. High standard deviations likely reflect differences in geographic origin, harvest time, and processing conditions. Of note, many of our tSGAs varied by up to three orders of magnitude among different analytes and tomato products. This finding indicates a broad range of tSGA concentrations in tomato-based products.

Alpha-tomatine, the first tSGA in the biosynthesis pathway, was found to be in the highest concentration in processed tomato products such as paste, pasta sauce, and soup (Table 5). The discrepancy between fresh and whole peeled tomatoes is hypothesized to be due to genetic and environmental conditions that influenced the chemical profile of the tomatoes prior to processing. Analyte groups like dehydrolycoperoside F, G or A, lycoperosides F, G, or esculeoside A and acetoxytomatine (commonly referred to as lycoperosides A, B, or C) were not detectable in most tomato products except for some fresh varieties and ketchup. Interestingly, lycoperosides F, G, or esculeoside A are typically the most abundant tSGA in fresh tomatoes (Iijima et al., 2013), though there is reported variation among different cultivars (Baldina et al., 2016). This observation raises questions about the effects of processing on tSGAs where few studies have been conducted to date (Tomas et al., 2017). While the chemically analogous potato glycoalkaloids are considered to be heat stable, high temperatures, pressures, and any combination thereof might be detrimental to some tSGAs or cause shifts in chemical profiles.

Concentrations of tSGAs in tomato products were normalized for serving size to contextualize how much might be ingested in a given meal. Other tomato phytochemicals, such as lycopene, tend to be found in concentrations ranging from 0.09 to $9.93 \mathrm{mg} / 100 \mathrm{~g}$ FW in fresh tomatoes (Dzakovich et al., 2019). Compared to major carotenoids found in tomato, tSGA concentrations were comparable (0.2 to $3.4 \mathrm{mg} /$ serving) (Cooperstone, 2020). This finding contradicts a long-standing misconception that tSGAs are degraded during ripening (Friedman, 2002). Rather, tSGAs such as alpha-tomatine are biochemically transformed during ripening 
TABLE 5 | Survey of tSGAs in common tomato-based products reported in $\mu \mathrm{g}$ per serving size.

\begin{tabular}{|c|c|c|c|c|c|c|c|c|}
\hline Analyte & $\begin{array}{l}\text { Fresh market } \\
\qquad(n=7)\end{array}$ & $\begin{array}{l}\text { Juice } \\
(n=3)\end{array}$ & $\begin{array}{l}\text { Ketchup } \\
(n=3)\end{array}$ & $\begin{array}{l}\text { Pasta sauce } \\
\qquad(n=3)\end{array}$ & $\begin{array}{l}\text { Paste } \\
(n=3)\end{array}$ & $\begin{array}{l}\text { Pizza sauce } \\
\qquad(n=3)\end{array}$ & $\begin{array}{l}\text { Soup } \\
(n=3)\end{array}$ & $\begin{array}{l}\text { Whole peeled } \\
\qquad(n=3)\end{array}$ \\
\hline Serving size $(g)$ : & 126 & 228.5 & 17 & 126 & 33 & 62 & 126 & 126 \\
\hline Esculeoside B & $4.3 \pm 9.7^{a}$ & $3.3 \pm 3.00$ & $0.3 \pm 0.6$ & $5.9 \pm 7.2$ & $3.6 \pm 0.8$ & $1.8 \pm 0.6$ & $2.0 \pm 2.1$ & $21.8 \pm 10.3$ \\
\hline Hydroxytomatine & $297.9 \pm 248.1$ & $54.3 \pm 25.0$ & $12.1 \pm 1.3$ & $80.4 \pm 14.5$ & $57.9 \pm 13.2$ & $26.4 \pm 4.9$ & $42.0 \pm 7.3$ & $50.4 \pm 3.7$ \\
\hline $\begin{array}{l}\text { Dehydrolyco- } \\
\text { peroside } \\
\text { F, G, or } \\
\text { Dehydroesculeoside }\end{array}$ & $7.0 \pm 12.1$ & N.D. ${ }^{b}$ & N.D. & N.D. & N.D. & N.D. & N.D. & N.D. \\
\hline $\begin{array}{l}\text { Lycoperoside F, } \\
\text { G or } \\
\text { Esculeoside A }\end{array}$ & $1589.4 \pm 1738.3$ & N.D. & N.D. & N.D. & N.D. & N.D. & N.D. & N.D. \\
\hline Acetoxytomatine & $30.6 \pm 31.6$ & $17.4 \pm 8.6$ & $3.1 \pm 2.0$ & $20.3 \pm 15.8$ & $25.0 \pm 4.1$ & $9.4 \pm 3.0$ & $10.0 \pm 4.8$ & $1.8 \pm 3.2$ \\
\hline Dehydrotomatine & $4.1 \pm 3.0$ & $41.0 \pm 29.0$ & $5.7 \pm 0.7$ & $41.3 \pm 14.9$ & $28.2 \pm 3.9$ & $19.4 \pm 2.0$ & $31.6 \pm 7.8$ & $11.3 \pm 5.9$ \\
\hline Alpha-tomatine & $64.5 \pm 56.0$ & $1083.5 \pm 747.4$ & $156.3 \pm 9.7$ & $1109.9 \pm 390.8$ & $889.5 \pm 119.4$ & $524.7 \pm 85.5$ & $964.3 \pm 62.5$ & $338.4 \pm 156.5$ \\
\hline Tomatidine & N.Q. ${ }^{c}$ & N.Q. & $0.4 \pm 0.3$ & $1.7 \pm 1.3$ & $0.8 \pm 0.0$ & $0.8 \pm 0.1$ & $1.5 \pm 0.5$ & $0.42 \pm 0.2$ \\
\hline Dehydrotomatidine & N.Q. & N.Q. & N.Q. & $0.2 \pm 0.1$ & $0.1 \pm 0.0$ & N.Q. & N.Q. & N.Q. \\
\hline Total & $3376.0 \pm 2886.3$ & $1307.7 \pm 823.7$ & $191.7 \pm 23.4$ & $1541.9 \pm 410.3$ & $1135.1 \pm 285.9$ & $736.5 \pm 166.6$ & $1126.3 \pm 34.4$ & $1101.3 \pm 116.5$ \\
\hline
\end{tabular}

${ }^{a}$ Mean \pm standard deviation. ${ }^{b}$ Not detected. ${ }^{c}$ Not quantified.

into glycosylated and acetylated forms. Overall, our methods were able to efficiently extract and analyze many types of tSGAs and generate the first quantitative concentration reports of these analytes in commonly consumed tomato products. Moreover, we found that tSGAs can be found in similar concentrations to other major phytochemicals in tomatoes such as carotenoids.

We have developed and described the first comprehensive extraction and analysis method for tSGAs. Our extraction method was able to quickly and efficiently extract tSGAs and allowed for high-throughput workflows (16 samples per $\sim 20 \mathrm{~min}$ ) to be utilized. Our UHPLC-MS/MS method was able to separate and quantify 18 tSGAs representing 9 different tSGA masses, as well as two internal standards, in $13 \mathrm{~min}$. Limits of quantification for commercially available tSGAs were 1.09 and 0.34 femtomoles on column for alpha-tomatine and tomatidine, respectively. This corresponds to 0.8 and $0.25 \mu \mathrm{g} / 100 \mathrm{~g}$ of alpha-tomatine and tomatidine in tomato, respectively, given our extraction procedures. Relative quantification for tSGAs and aglycones that did not have commercially available standards was performed using alpha-tomatine and tomatidine, respectively. Our methods were able to successfully profile tSGAs in a comprehensive array of commonly available tomato-based products. These values are among the first to be reported in the literature and can serve as benchmarks for future studies investigating tSGAs in a variety of contexts. Our extraction and UHPLC-MS/MS method will allow researchers to rapidly and accurately generate data about tSGAs enabling their continued study.

\section{DATA AVAILABILITY STATEMENT}

The raw data supporting the conclusions of this article will be made available by the authors, without undue reservation, to any qualified researcher.

\section{AUTHOR CONTRIBUTIONS}

MD and JC contributed to the project ideation. MD contributed to the method development and drafted the manuscript. MD and $\mathrm{JH}$ analyzed samples. $\mathrm{MD}, \mathrm{JH}$, and $\mathrm{JC}$ analyzed and interpreted the data, and edited the manuscript. All authors have read and approved the final manuscript. JC has responsibility for final content.

\section{FUNDING}

Financial support was provided by the USDA-NIFA National Needs Fellowship (2014-38420-21844), USDA Hatch funds (OHO01470), Foods for Health, a focus area of the Discovery Themes Initiative at The Ohio State University, and the Ohio Agricultural Research and Development Center.

\section{ACKNOWLEDGMENTS}

We thank David Francis, Jiheun Cho, Troy Aldrich (The Ohio State University, Ohio Agriculture Research and Development Center), and the North Central Agricultural Research Station crews for assistance with selecting, planting, and harvesting tomatoes used in this study. This manuscript has been released as a pre-print at bioRxiv https://www.biorxiv.org/content/10.1101/ 2019.12.23.878223v1.

\section{SUPPLEMENTARY MATERIAL}

The Supplementary Material for this article can be found online at: https://www.frontiersin.org/articles/10.3389/fpls.2020.00767/ full\#supplementary-material 


\section{REFERENCES}

Alseekh, S., Tohge, T., Wendenberg, R., Scossa, F., Omranian, N., Li, J., et al. (2015). Identification and mode of inheritance of quantitative trait loci for secondary metabolite abundance in tomato. Plant Cell 27, 485-512. doi: 10.1105/tpc.114. 132266

Baldina, S., Picarella, M. E., Troise, A. D., Pucci, A., Ruggieri, V., Ferracane, R., et al. (2016). Metabolite profiling of italian tomato landraces with different fruit types. Front. Plant Sci. 7:664. doi: 10.3389/fpls.2016.00664

Ballester, A.-R., Tikunov, Y., Molthoff, J., Grandillo, S., Viquez-Zamora, M., de Vos, R., et al. (2016). Identification of loci affecting accumulation of secondary metabolites in tomato fruit of a solanum lycopersicum $\times$ solanum chmielewskii introgression line population. Front. Plant Sci. 7:1428. doi: 10.3389/fpls.2016. 01428

Caprioli, G., Cahill, M. G., and James, K. J. (2014). Mass fragmentation studies of $\alpha$-tomatine and validation of a liquid chromatography LTQ orbitrap mass spectrometry method for its quantification in tomatoes. Food Anal. Methods 7, 1565-1571. doi: 10.1007/s12161-013-9771-9

Cárdenas, P. D., Sonawane, P. D., Heinig, U., Bocobza, S. E., Burdman, S., and Aharoni, A. (2015). The bitter side of the nightshades: genomics drives discovery in solanaceae steroidal alkaloid metabolism. Phytochemistry 113, 24-32. doi: 10.1016/j.phytochem.2014.12.010

Cárdenas, P. D., Sonawane, P. D., Pollier, J., Vanden Bossche, R., Dewangan, V., Weithorn, E., et al. (2016). GAME9 regulates the biosynthesis of steroidal alkaloids and upstream isoprenoids in the plant mevalonate pathway. Nat. Commun. 7:10654. doi: 10.1038/ncomms10654

Cayen, M. N. (1971). Effect of dietary tomatine on cholesterol metabolism in the rat. J. Lipid Res. 12, 482-490.

Choi, S. H., Ahn, J.-B., Kozukue, N., Kim, H.-J., Nishitani, Y., Zhang, L., et al. (2012). Structure-activity relationships of $\alpha_{-}, \beta_{1}-, \gamma$-, and $\delta$-tomatine and tomatidine against human breast (MDA-MB-231), gastric (KATO-III), and prostate (PC3) cancer cells. J. Agricult. Food Chem. 60, 3891-3899. doi: 10.1021/ jf3003027

Chungcharoen, A. (1988). Glycoalkaloid Content Of Potatoes Grown Under Controlled Environments And Stability Of Glycoalkaloids During Processing. $\mathrm{Ph}$. D. Thesis, University of Wisconsin, Madison, WI.

Cichon, M. J., Riedl, K. M., Wan, L., Thomas-Ahner, J. M., Francis, D. M., Clinton, S. K., et al. (2017). Plasma metabolomics reveals steroidal alkaloids as novel biomarkers of tomato intake in mice. Mol. Nutr. Food Res. 61:1700241. doi: $10.1002 / \mathrm{mnfr} .201700241$

Cooperstone, J. L. (2020). "Lycopene," in Handbook of Nutraceuticals and Functional Foods, eds E. C. Wildman and R. S. Bruno (Boca Raton, FL: CRC Press), 37-53. doi: 10.1201/9780429195594-3

Cooperstone, J. L., Tober, K. L., Riedl, K. M., Teegarden, M. D., Cichon, M. J., Francis, D. M., et al. (2017). Tomatoes protect against development of UVinduced keratinocyte carcinoma via metabolomic alterations. Sci. Rep. 7:5106. doi: 10.1038/s41598-017-05568-7

Del Giudice, R., Raiola, A., Tenore, G. C., Frusciante, L., Barone, A., Monti, D. M., et al. (2015). Antioxidant bioactive compounds in tomato fruits at different ripening stages and their effects on normal and cancer cells. J. Funct. Foods 18, 83-94. doi: 10.1016/J.JFF.2015.06.060

Dzakovich, M. P., Gas-Pascual, E., Orchard, C. J., Sari, E. N., Riedl, K. M., Schwartz, S. J., et al. (2019). Analysis of tomato carotenoids: comparing extraction and chromatographic methods. J. AOAC Int. 102, 1069-1079. doi: 10.5740/jaoacint. 19-0017

Eltayeb, E. A., and Roddick, J. G. (1984). Changes in the alkaloid content of developing fruits of tomato (Lycopersicon esculentum Mill .) II. J. Exp. Bot. 35, 261-267. doi: 10.1093/jxb/35.2.261

Etalo, D. W., De Vos, R. C. H., Joosten, M. H. A. J., and Hall, R. D. (2015). Spatially resolved plant metabolomics: some potentials and limitations of laser-ablation electrospray ionization mass spectrometry metabolite imaging. Plant Physiol. 169, 1424-1435. doi: 10.1104/pp.15.01176

Fontaine, T. D., Irving, G. W., Ma, R., Poole, J. B., and Doolittle, S. P. (1948). Isolation and partial characterization of crystalline tomatine, an antibiotic agent from the tomato plant. Arch. Biochem. 18, 467-475.

Friedman, M. (2002). Tomato glycoalkaloids: role in the plant and in the diet. J. Agricult. Food Chem. 50, 5751-5780. doi: 10.1021/jf020560c
Friedman, M., and Levin, C. E. (1992). Reversed-phase high-performance liquid chromatographic separation of potato glycoalkaloids and hydrolysis products on acidic columns. J. Agricult. Food Chem. 40, 2157-2163. doi: 10.1021/ jf00023a023

Friedman, M., and Levin, C. E. (1998). Dehydrotomatine content in tomatoes. J. Agricult. Food Chem. 46, 4571-4576. doi: 10.1021/jf9804589

Fujiwara, Y., Takaki, A., Uehara, Y., Ikeda, T., Okawa, M., Yamauchi, K., et al. (2004). Tomato steroidal alkaloid glycosides, esculeosides A and B, from ripe fruits. Tetrahedron 60, 4915-4920. doi: 10.1016/j.tet.2004.03.088

Hövelmann, Y., Jagels, A., Schmid, R., Hübner, F., and Humpf, H.-U. (2019). Identification of potential human urinary biomarkers for tomato juice intake by mass spectrometry-based metabolomics. Eur. J. Nutr. 4, 1-13. doi: 10.1007/ s00394-019-01935-4

Iijima, Y., Fujiwara, Y., Tokita, T., Ikeda, T., Nohara, T., Aoki, K., et al. (2009). Involvement of ethylene in the accumulation of esculeoside a during fruit ripening of tomato (Solanum lycopersicum). J. Agricult. Food Chem. 57, 32473252. doi: 10.1021/jf8037902

Iijima, Y., Nakamura, Y., Ogata, Y., Tanaka, K. K., Sakurai, N., Suda, K., et al. (2008). Metabolite annotations based on the integration of mass spectral information. Plant J. Cell Mol. Biol. 54, 949-962. doi: 10.1111/j.1365-313X.2008. 03434.X

Iijima, Y., Watanabe, B., Sasaki, R., Takenaka, M., Ono, H., Sakurai, N., et al. (2013). Steroidal glycoalkaloid profiling and structures of glycoalkaloids in wild tomato fruit. Phytochemistry 95, 145-157. doi: 10.1016/J.PHYTOCHEM.2013.07.016

Irving, G. W., Fontaine, T. D., and Doolittle, S. P. (1945). Lycopersicin, a fungistatic agent from the tomato plant. Science 102, 9-11. doi: 10.1126/science.102.2636.9

Itkin, M., Heinig, U., Tzfadia, O., Bhide, A. J., Shinde, B., Cardenas, P. D., et al. (2013). Biosynthesis of antinutritional alkaloids in solanaceous crops is mediated by clustered genes. Science (New York N.Y.) 341, 175-179. doi: $10.1126 /$ science. 1240230

Itkin, M., Rogachev, I., Alkan, N., Rosenberg, T., Malitsky, S., Masini, L., et al. (2011). Glycoalkaloid metabolism1 is required for steroidal alkaloid glycosylation and prevention of phytotoxicity in tomato. Plant Cell 23, 45074525. doi: 10.1105/tpc.111.088732

Keukens, E. A. J., Hop, M. E. C. M., and Jongen, W. M. F. (1994). Rapid high-performance liquid chromatographic method for the quantification oftomatine in tomato. J. Agric. Food Chem. 42, 2475-2477. doi: 10.1021/ jf00047a020

Kopec, R. E., Cooperstone, J. L., Cichon, M. J., and Schwartz, S. J. (2012). “Analysis Methods of Carotenoids," in Analysis of Antioxidant-Rich Phytochemicals, eds Z. Xu and R. Luke (Oxford: Wiley-Blackwell), 105-148. doi: 10.1002/ 9781118229378.ch4

Kozukue, N., and Friedman, M. (2003). Tomatine, chlorophyll, Beta-carotene and lycopene content in tomatoes during growth and maturation. J. Sci. Food Agricult. 83, 195-200. doi: 10.1002/jsfa.1292

Kozukue, N., Han, J.-S., Lee, K.-R., and Friedman, M. (2004). Dehydrotomatine and $\alpha$-tomatine content in tomato fruits and vegetative plant tissues. J. Agricult. Food Chem. 52, 2079-2083. doi: 10.1021/jf0306845

Lawson, D. R., Erb, W. A., and Miller, A. R. (1992). Analysis of solanum alkaloids using internal standardization and capillary gas chromatography. Food Chem. 40, 2186-2191. doi: 10.1021/jf00023a028

Lee, K.-R., Kozukue, N., Han, J.-S., Park, J.-H., Chang, E., Baek, E.-J., et al. (2004) Glycoalkaloids and metabolites inhibit the growth of human colon (HT29) and Liver (HepG2) cancer cells. J. Agricult. Food Chem. 52, 2832-2839. doi: $10.1021 /$ jf030526d

Manabe, H., Fujiwara, Y., Ikeda, T., Ono, M., Murakami, K., Zhou, J. R., et al. (2013). Saponins esculeosides B-1 and B-2 in Italian canned tomatoes. Chem. Pharmaceut. Bull. 61, 764-767. doi: 10.1248/cpb.c13-00202

Mintz-Oron, S., Mandel, T., Rogachev, I., Feldberg, L., Lotan, O., Yativ, M., et al. (2008). Gene expression and metabolism in tomato fruit surface tissues. Plant Physiol. 147, 823-851. doi: 10.1104/pp.108.116004

Moco, S., Bino, R. J., Vorst, O., Verhoeven, H. A., de Groot, J., van Beek, T. A., et al. (2006). A liquid chromatography-mass spectrometry-based metabolome database for tomato. Plant physiology 141, 1205-1218. doi: 10.1104/pp.106. 078428

Nohara, T., Fujiwara, Y., Zhou, J. R., Urata, J., Ikeda, T., Murakami, K., et al. (2015). Saponins, esculeosides B-1 and B-2, in tomato juice and sapogenol, 
esculeogenin B 1. Chem. Pharmaceut. Bull. 63, 848-850. doi: 10.1248/cpb.c1500449

Ökmen, B., Etalo, D. W., Joosten, M. H. A. J., Bouwmeester, H. J., de Vos, R. C. H., Collemare, J., et al. (2013). Detoxification of $\alpha$-tomatine by Cladosporium fulvum is required for full virulence on tomato. New Phytol. 198, 1203-1214. doi: $10.1111 / \mathrm{nph} .12208$

Ono, H., Kozuka, D., Chiba, Y., Horigane, A., and Isshiki, K. (1997). Structure and Cytotoxicity of Dehydrotomatine, a Minor Component of Tomato Glycoalkaloids. J. Agricult. Food Chem. 45, 3743-3746. doi: 10.1021/jf970 $253 \mathrm{k}$

Rick, C. M., Uhlig, J. W., and Jones, D. (1994). High alpha-tomatine content in ripe fruit of Andean Lycopersicon esculentum var. cerasiforme: developmental and genetic aspects. Proc. Natl. Acad. Sci. U.S.A. 91, 12877-12881. doi: 10.1073/ pnas.91.26.12877

Rodriguez-Saona, L. E., Wrolstad, R. E., and Pereira, C. (1999). Glycoalkaloid content and anthocyanin stability to alkaline treatment of red-fleshed potato extracts. J. Food Sci. 64, 445-450. doi: 10.1111/j.1365-2621.1999.tb1 5060.x

Schlösser, E., and Gottlieb, D. (1966). Mode of hemolytic action of the antifungal polyene antibiotic filipin. Zeitschr. Naturforsc. Sec. B J. Chem. Sci. 21, 74-77. doi: 10.1515/znb-1966-0120

Sonawane, P. D., Heinig, U., Panda, S., Gilboa, N. S., Yona, M., Pradeep, K. S., et al. (2018). Short-chain dehydrogenase/reductase governs steroidal specialized metabolites structural diversity and toxicity in the genus Solanum. Proc. Natl. Acad. Sci. U.S.A. 115, E5419-E5428. doi: 10.1073/pnas.180483 5115
Tajner-Czopek, A., Rytel, E., Aniołowska, M., and Hamouz, K. (2014). The influence of French fries processing on the glycoalkaloid content in colouredfleshed potatoes. Eur. Food Res. Technol. 238, 895-904. doi: 10.1007/s00217014-2163-6

Tomas, M., Beekwilder, J., Hall, R. D., Sagdic, O., Boyacioglu, D., and Capanoglu, E. (2017). Industrial processing versus home processing of tomato sauce: Effects on phenolics, flavonoids and in vitro bioaccessibility of antioxidants. Food Chem. 220, 51-58. doi: 10.1016/j.foodchem.2016.09.201

Yamanaka, T., Vincken, J.-P., Zuilhof, H., Legger, A., Takada, N., and Gruppen, H. (2009). C22 isomerization in $\alpha$-tomatine-to-esculeoside a conversion during tomato ripening is driven by $\mathrm{C} 27$ hydroxylation of triterpenoidal skeleton. J. Agric. Food Chem. 57, 3786-3791. doi: 10.1021/jf900017n

Zhu, G., Wang, S., Huang, Z., Zhang, S., Liao, Q., Zhang, C., et al. (2018). Rewiring of the fruit metabolome in tomato breeding. Cell 172, 249-261.e12. doi: 10. 1016/j.cell.2017.12.019

Conflict of Interest: The authors declare that the research was conducted in the absence of any commercial or financial relationships that could be construed as a potential conflict of interest.

Copyright (c) 2020 Dzakovich, Hartman and Cooperstone. This is an open-access article distributed under the terms of the Creative Commons Attribution License (CC BY). The use, distribution or reproduction in other forums is permitted, provided the original author(s) and the copyright owner(s) are credited and that the original publication in this journal is cited, in accordance with accepted academic practice. No use, distribution or reproduction is permitted which does not comply with these terms. 